\title{
Protée
}

\section{Marcel Marois | La dialectique de l'ordre et du chaos}

Paul Bourassa

Volume 38, numéro 3, hiver 2010

Les concepts aux frontières du savoir contemporain

URI : https://id.erudit.org/iderudit/045617ar

DOI : https://doi.org/10.7202/045617ar

Aller au sommaire du numéro

Éditeur(s)

Département des arts et lettres - Université du Québec à Chicoutimi

ISSN

0300-3523 (imprimé)

1708-2307 (numérique)

Découvrir la revue

Citer cet article

Bourassa, P. (2010). Marcel Marois | La dialectique de l'ordre et du chaos. Protée, 38(3), 65-70. https://doi.org/10.7202/045617ar d'utilisation que vous pouvez consulter en ligne.

https://apropos.erudit.org/fr/usagers/politique-dutilisation/ 


\section{Marcel Marois}
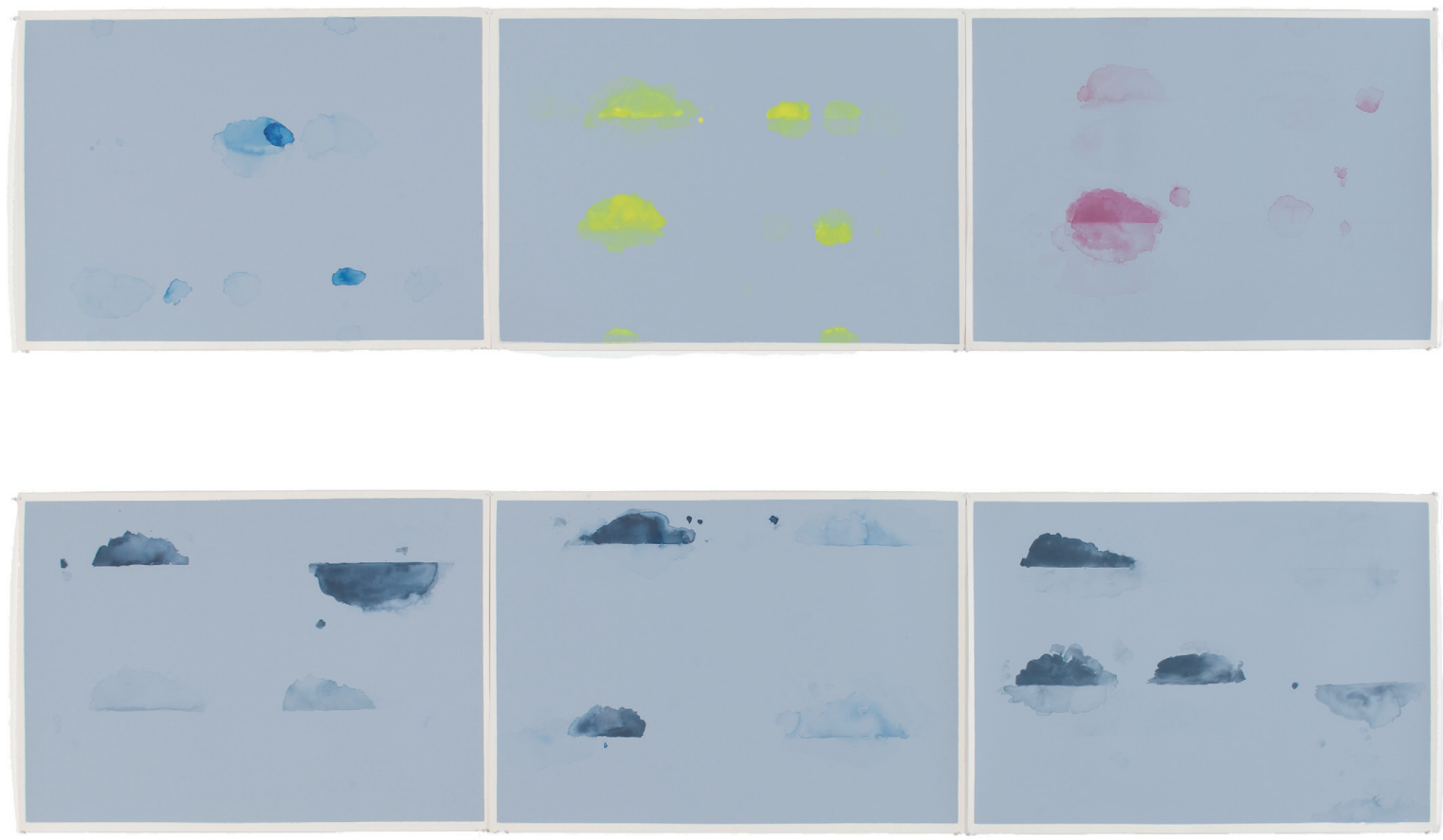

Suspension bleu, jaune, magenta, 2010.

Aquarelle sur papier Spectrum | 50 × $70 \mathrm{~cm}$ chacune.

Déplacement no 1, no 2, no 3, 2010.

Aquarelle sur papier Spectrum | 50 × $70 \mathrm{~cm}$ chacune.

Photographies de Yvan Binet. 


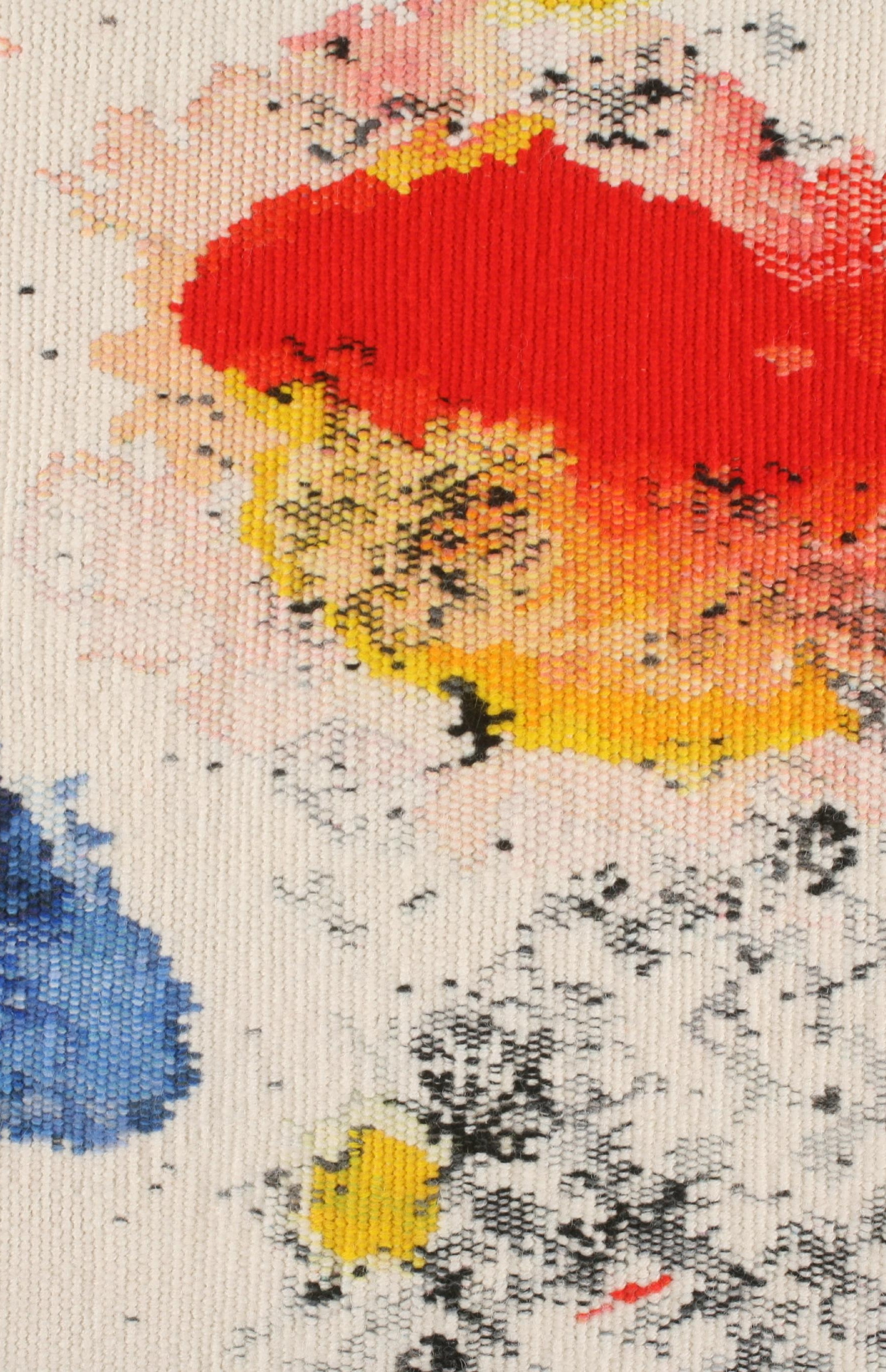




\title{
LA DIALECTIQUE DE L'ORDRE ET DU CHAOS
}

\author{
Une présentation de Paul Bourassa
}

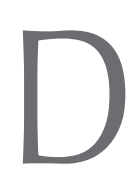

ANS SON TRAVAIL RÉCENT, Marcel Marois a délaissé le monde de la une journalistique, de la catastrophe écologique, qu'il transformait jusqu'alors par un travail de déconstruction et de reconstruction de l'image en des œuvres tissées qui donnaient à l'événement toute l'épaisseur du phénomène, c'est-à-dire qui se manifestent à la conscience et s'inscrivent dans des structures plus profondes qui sont de I'ordre de la longue durée. Ses œuvres récentes, deux tapisseries magistrales Averse chromatique (2008) et Double horizon (2010), mais également une série de dessins à l'aquarelle (les séries Contemplation, Perception, Silence de 2007-2008 et Suspension et Déplacement de 2010) ont franchi la «barrière » de l'abstraction, une tendance qui s'est matérialisée au tournant des années 2000. Cet abandon de toute référence au réel signifie-t-il pour autant que l'artiste a changé sa vision ou sa manière ? À notre sens, non. Autant dans ses œuvres figuratives des années 1990 que dans ses abstractions des années 2000, Marois travaille toujours selon une dialectique de l'ordre et du chaos. La théorie du chaos veut qu'une infime imprécision dans l'ordre des données de départ crée des perturbations imprévisibles dans le long terme. C'est la métaphore du battement d'aile d'un papillon au Brésil qui pourrait provoquer une tornade au Texas... Mais Marois a plutôt une démarche opposée. Il contrôle et stabilise la spontanéité et le hasard. Du chaos, il cherche l'ordre, de la tempête, il soutire l'intime vibration de l'air.

Le carton de tapisserie est le lieu où Marois laisse s'exprimer la couleur et le geste. L'aquarelle et la gouache jouent de transparence et d'opacité. Taches et coulures s'affirment. Mais déjà l'artiste y commence son travail de harnachement par collage et découpage, ajoutant, retranchant, reconfigurant son impulsion première. La composition, au final, n'est pas le résultat d'un automatisme, d'une improvisation, d'un chaos, mais une structure où des plans sont clairement définis, où les traces d'une manipulation de l'image influent sur le chromatisme et la nature même des taches de couleurs, où l'ordre déjà s'installe. Puis commence le long, le très long processus de tissage où chaque ton est reconstruit point par point, où chaque nuance est décomposée et rendue à l'aide de plusieurs fils de couleurs différentes.

Page de gauche | Averse chromatique (détail), 2008. Tapisserie de haute lice | laine | 256 × $305 \mathrm{~cm}$.

Photographie de Yvan Binet. 
On serait tenté d'établir un parallèle avec la photographie numérique où, à l'écran, les valeurs colorées sont obtenues pixel par pixel. Mais, chez Marois, il y a cette matérialité de la fibre, absente de l'imagerie informatique. La régularité de la trame du tissage est aussi le résultat d'un battement..

On retrouve dans les dessins récents de Marois cette même précision cachée, cette divine proportion dans la fragilité de la touche. La fluidité de l'aquarelle est contrôlée malgré son apparente indocilité, soit par l'aspect sériel de larges bandes parallèles où la couleur "s'épuise», soit par l'apparition d'horizontales qui traversent les taches, comme si ses ciels de couleurs étaient dotés de plusieurs horizons.

Chez Marcel Marois, la synthèse entre la turbulence de l'élan créateur et la cohérence $d^{\prime}$ 'une exécution totalement contrôlée s'opère parfaitement. De cette dialectique de l'ordre et du chaos émergent des œuvres qui ont la délicatesse de l'aile du papillon et la force de I'ouragan. 


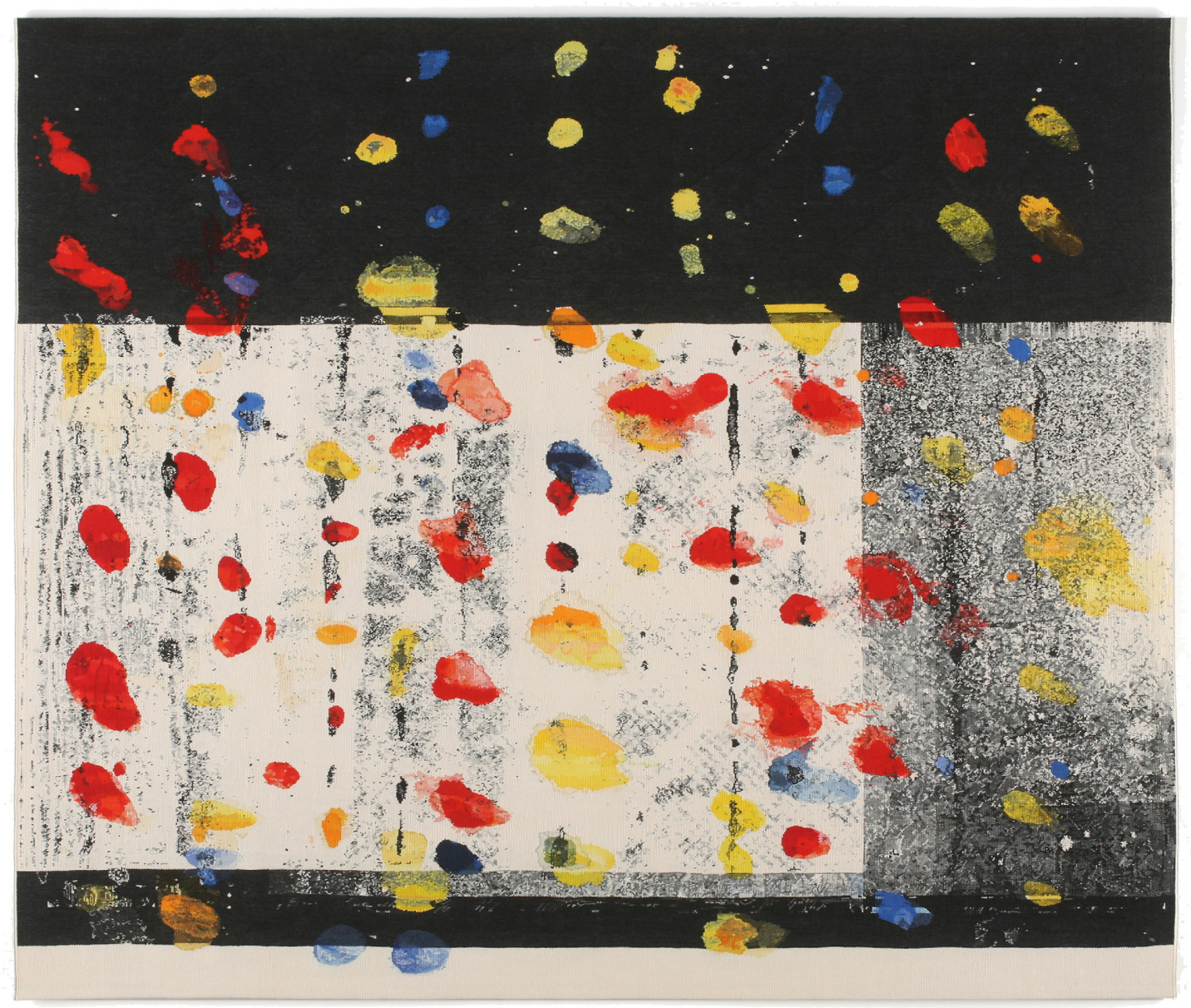

Averse chromatique, 2008.

Tapisserie de haute lice | laine | 256 × $305 \mathrm{~cm}$.

Photographie de Yvan Binet. 


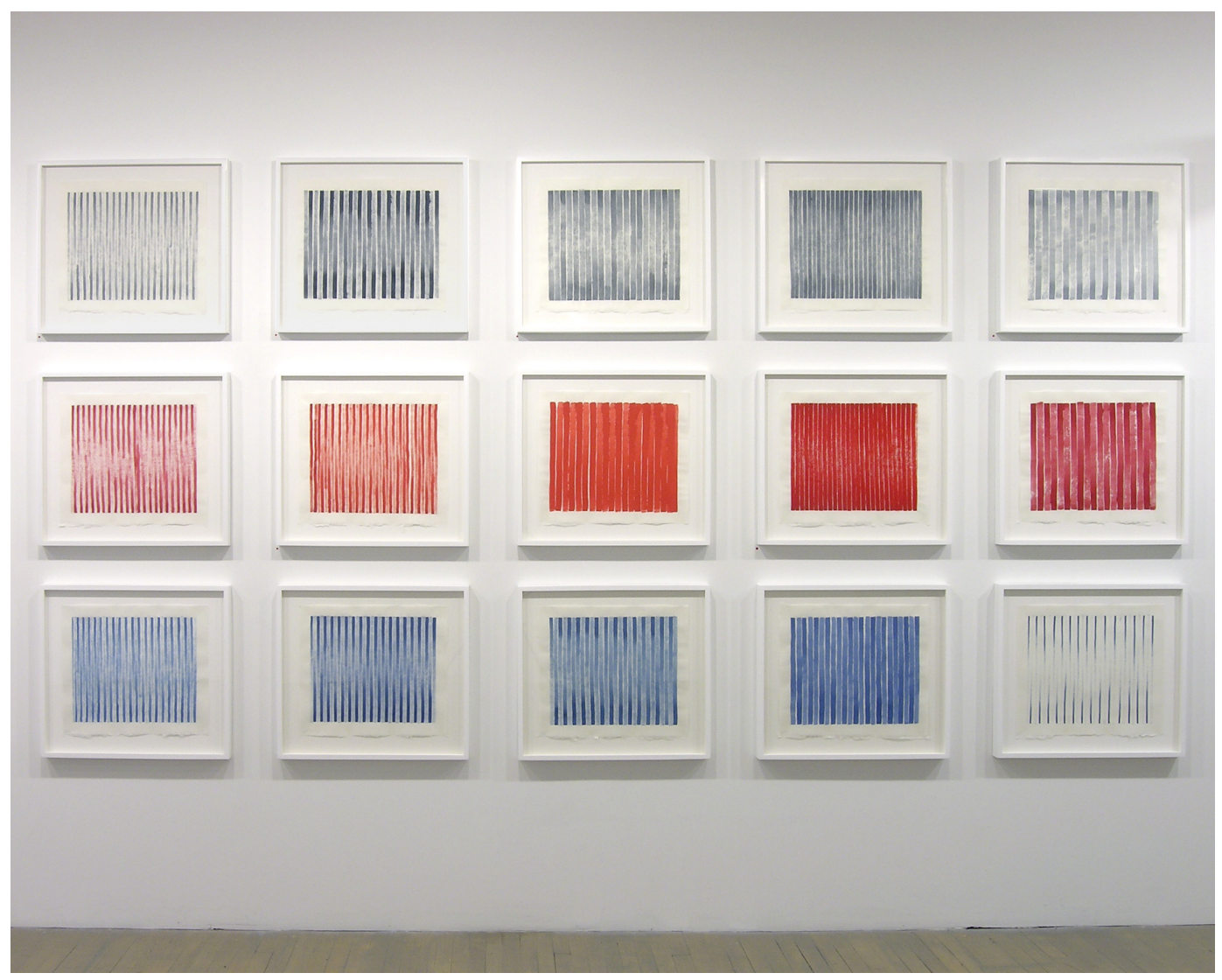

Série Contemplation, Série Perception, Série Silence, 2007-2008.

Aquarelles sur papier | 42,5 x 47,5 cm chacune.

Photographie de Christian Lambert | Galerie Roger Bellemare. 